\title{
The role of endoscopy after upper gastrointestinal bleeding in sub-Saharan Africa: A prospective observational cohort study
}

\author{
Jared R. Gallaher ${ }^{1}$, Gift Mulima ${ }^{2}$, Javeria Qureshi ${ }^{1}$, Carol G. Shores ${ }^{2,3}$, Anthony G. Charles ${ }^{1,2}$ \\ 1. Department of Surgery, University of North Carolina School of Medicine, CB\# 7228, Chapel Hill, NC, USA \\ 2. Department of Surgery, Kamuzu Central Hospital, Lilongwe, Malawi \\ 3. Department of Otolaryngology/Head \& Neck Surgery University of North Carolina School of Medicine, CB\#7070, Chapel Hill, NC, USA
}

Corresponding author: Jared R. Gallaher; Jared.gallaher@gmail.com

Background
Upper gastrointestinal (UGI) bleed is a common surgical disease in sub-Saharan Africa where there is often a lack of diagnostic and
interventional adjuncts such as endoscopy. This study sought to characterize the role of endoscopy in management of acute UGI
bleeding.
Materials and Methods
This is a prospective observational analysis of adults presenting with an UGI bleed to a tertiary center in Lilongwe, Malawi, over two
years. Patients were classified as having no endoscopy, diagnostic endoscopy, or endoscopy with variceal banding. Bivariate, survival
analysis, and logistic regression analyses were used to compare intervention cohorts.
Results
293 patients were included with 49 patients $(16.7 \%)$ receiving endoscopy with banding, 65 (22.2\%) patients receiving diagnostic
endoscopy only, and $179(61.1 \%)$ receiving no endoscopy. Upon survival analysis comparing to the no endoscopy group, cox hazard
modelling showed an adjusted hazard ratio over 30 days of $0.12(95 \%$ CI $0.02,0.88, p=0.038)$ for the endoscopic banding group and
a hazard ratio of 0.39 ( $95 \%$ CI $0.13,1.16, \mathrm{p}=0.090)$ for the diagnostic endoscopy only group. Physical exam findings consistent with
cirrhosis and decreasing age were independent predictors of an endoscopic diagnosis of variceal bleeding.
Conclusion
Esophagogastric varices are a common cause of UGI bleeding in sub-Saharan Africa and can be predicted with age and physical exam
findings. Endoscopy with variceal banding has a survival benefit for patients presenting with acute UGI bleed even with relatively
low utilization. Appropriately triaging patients with likely variceal bleeding and improving endoscopy capacity would likely have a
significant impact on mortality.
Keywords: endoscopy, upper gastrointestinal bleeding, sub-Saharan Africa

\section{Introduction}

The burden of surgical disease in sub-Saharan African currently exceeds the capacity for surgical care delivery. ${ }^{1}$ This is especially evident when multidisciplinary coordination and complex health system utilization is required, as with acute upper gastrointestinal (UGI) bleeding. A patient with acute UGI bleeding requires monitored resuscitation, timely transfusion services, endoscopic diagnostic and therapeutic adjuncts with available expertise, appropriate pharmacotherapy, and possibly surgical intervention. ${ }^{2,3}$

In sub-Saharan Africa, the etiopathogenesis of UGI bleeding is heterogeneous with significant regional variation. ${ }^{4-11}$ In contrast to the United States and other high-income countries, esophagogastric varices (not peptic ulcer disease) are the predominant cause of UGI bleeding in many regions. ${ }^{6-11}$ Esophagogastric varices are often caused by liver cirrhosis, which has increased in incidence in this region. The burden of liver cirrhosis rose by $57 \%$ over the last two decades and liver cirrhosis-related mortality doubled from 1980 through 2010 throughout sub-Saharan Africa. ${ }^{12}$ This is attributable to the high prevalence of Hepatitis B and C, alcohol use, and other regional pathologies such as schistosomiasis, which is endemic in Malawi. ${ }^{12,13}$ The data that the burden of liver cirrhosis is increasing substantially in sub-Saharan Africa combined with evidence that esophagogastric bleeding is also increasing in incidence indicates this is an important public health issue. ${ }^{6}$

Our experience at a tertiary center in Malawi suggests an increase over time in the number of patients with esophagogastric variceal bleeding but there is a dearth of data on the clinical characteristics and outcomes in this patient population in sub-Saharan Africa. Similarly, resource constraints further impact the utility of diagnostic adjuncts and clinical management. When diagnostic and therapeutic interventions are scarce, providers must endeavour to appropriately triage patients who are most likely to benefit from any available therapeutic intervention. In this study, we sought to describe the role of endoscopy in patients with acute UGI bleeding presenting to our center in Malawi and identify patient cohorts who would benefit most from diagnostic and therapeutic intervention.

\section{Methods}

This was a prospective observational study of adult patients presenting to Kamuzu Central Hospital $(\mathrm{KCH})$ with UGI bleeding from October 2011 to October 2013. KCH is a public 600-bed tertiary care hospital in the capital city of Lilongwe, which serves as a referral center for approximately 5 million people in the central region of Malawi. $\mathrm{KCH}$ is equipped with two esophagogastroduodenoscopes and two colonoscopes. 
Patients were prospectively enrolled if $\geq 18$ years old, presented within 24 hours of developing hematemesis or passage of melanotic stools, and required hospital admission. Patients arriving at the hospital with UGI bleed in extremis were not enrolled. Baseline demographic and clinical data were obtained including presenting symptomatology, physical examination findings, vital signs, laboratory investigation results, and clinical outcome (lived/died). Post-discharge follow-up was obtained in clinic or via telephone.

The primary outcome measure was mortality. The primary intervention for this analysis was esophagogastroduodenoscopy (EGD). Patients were categorized into three groups: (1) those that received no intervention, (2) diagnostic endoscopy only, and (3) diagnostic and therapeutic endoscopy (variceal banding). This is an observational study and all clinical interventions including the decision to schedule endoscopy and the use of variceal banding were made by the local clinicians based on clinical presentation and resource availability. $\mathrm{KCH}$ does not have the resources to perform emergent endoscopy.

We initially examined the characteristics of the sample population assessing the distribution of the variables, and any impact of missing data or extreme values. The mean, standard deviation, and the shape of the distribution were calculated for each continuous variable, and frequencies were tabulated for categorical variables. To identify potential confounders, we examined the equality of distribution of independent variables between the three groups, comparing means between groups for the continuous variables or percentages for the categorical variables. Bivariate analysis for each independent variable based on mortality outcome was performed using Pearson's correlation for the categorical variables, and 2-sample t-tests or one-way analysis of variance for continuous variables. For continuous variables with non-parametric distributions, we used a Kruskal-Wallis test to compare medians. Means are reported with standard deviations $(\underline{ \pm})$.

We used survival analysis to estimate the hazard ratio for inhospital mortality at thirty-days for both the diagnostic only group and the therapeutic endoscopy with variceal banding group compared to the reference group (no endoscopy group). Because there were no deaths in the therapeutic endoscopy group, we used an imputation strategy that allowed us to calculate a hazard ratio. A patient death was imputed in all three study groups with patient characteristics reflective of the mean of that respective study group. For categorical variables, each imputed patient was given the same characteristic variable value. We chose variable values that would give the most conservative model estimate of the adjusted hazard ratio. We initially plotted an unadjusted Kaplan-Meier curve comparing the three patient groups and then utilized a Cox regression model to estimate the mortality hazard ratio between the three patient groups, adjusting for confounders. Among potential confounders, we accessed and corrected for violation of the proportional hazards assumption. A potential confounder was included in the model if it substantially affected the adjusted hazard ratio. An adjusted hazard ratio and $95 \%$ confidence interval compared to the referent is reported for both intervention groups.

Finally, among the intervention arms, diagnostic only and diagnostic with therapeutic endoscopy, we used bivariate analysis to compare those with a positive endoscopic diagnosis of variceal bleeding and those with another endoscopic diagnosis. Physical exam findings consistent with cirrhosis or portal hypertension were defined as the presence of one or more of the following: ascites, jaundice, hepatomegaly, or splenomegaly. Ultrasound findings consistent with cirrhosis or portal hypertension were defined as the presence of one or more of the following: ascites, hepatomegaly, splenomegaly, or cirrhotic liver. Multivariate logistic regression modelling was used to examine predictors of endoscopically confirmed variceal bleeding. All clinically relevant variables were initially included. Variables were removed and reduced models were compared with a more complete, adjusted model using a maximum likelihood ratio test. If there was no statistically significant difference between models, those variables were excluded in the final predictive model. Odds ratios and adjusted predicted probabilities with $95 \%$ confidence intervals are reported using the final model.

All statistical analysis was performed using Stata/SE 13.1 (Stata- Corp LP, College Station, TX). The University of North Carolina Institutional Review Board (Study \# 11-1649) and the Malawi National Health Services Review Committee (Protocol \#891) approved this study, and consents were obtained from the patient or their relative if they were not able to sign the consent.

\section{Results}

The study enrolled 293 patients with 179 patients (61.1\%) in the control group, receiving no endoscopy. Among the remaining patients, $65(22.2 \%)$ received diagnostic endoscopy only, and 49 patients $(16.7 \%)$ received therapeutic endoscopy with banding. There was no statistically significant difference in the mean age between the three patient groups, with a mean of $41.6 \pm 16.1,43.7 \pm 17.0$, and $40.2 \pm 12.6$ years for the control group, diagnostic endoscopy only and the therapeutic endoscopy, respectively $(\mathrm{p}=0.08)$ (Table 1$)$. All three groups had a male preponderance $(63.1 \%, 64.6 \%, 51.0 \%, \mathrm{p}=0.051)$. Clinical history was statistically similar among all the three groups. There were more patients in the banding group with a previous history of upper GI bleeding $(35.8 \%, 32.3 \%$, $44.9 \%, \mathrm{p}=0.62)$ but with fewer alcohol users $(40.2 \%, 40.0 \%$, $26.5 \%, \mathrm{p}=0.41)$ or smokers $(27.9 \%, 26.2 \%, 14.3 \%, \mathrm{p}=0.26)$. Nearly every patient presented with hematemesis $(96.7 \%$, $96.9 \%, 95.9 \%, \mathrm{p}=0.96)$ and a majority also had melena $(64.8 \%, 69.2 \%, 71.4 \%, \mathrm{p}=0.77)$.

Mean presenting heart rate was clinically similar among the three groups $(100.0 \pm 19.6 \mathrm{bpm}, 95.4 \pm 16.8 \mathrm{bpm}, 97.7 \pm 25.0$ bpm, $\mathrm{p}=0.029)$ as was mean systolic blood pressure (106.7 $\pm 18.6 \mathrm{mmHg}, 108.7 \pm 21.8 \mathrm{mmHg}, 103.3 \pm 18.5 \mathrm{mmHg}$, $\mathrm{p}=0.29$ ). Patients who received either diagnostic endoscopy or endoscopy with banding were more likely to have their hemoglobin $(48.6 \%, 70.8 \%, 77.5 \%, \mathrm{p}<0.001)$ or platelet level $(29.6 \%, 38.5 \%, 57.1 \%, \mathrm{p}=0.002)$ measured at admission. Among those who had their hemoglobin measured at admission, there was a statistically significant difference in mean hemoglobin $(\mathrm{g} / \mathrm{dL})$ at $7.2 \pm 3.3,8.1 \pm 4.1$, and $6.2 \pm 2.2$ $(\mathrm{p}=0.001)$ for the three groups, respectively. Mean platelet count $\left(\times 10^{\circ} / \mathrm{L}\right)$ was lower in the banding group compared to the other two groups at $133.7 \pm 123.0,253.0 \pm 214.6$, and $91.5 \pm 111.3(\mathrm{p}<0.001)$, respectively.

Besides endoscopy, other common interventions used were blood product transfusions and pharmacotherapy including enteral propranolol after bleeding had stopped, omeprazole, spironolactone, and diuretics. In terms of transfusion, 209 $(71.3 \%)$ patients were transfused with at least one unit of Https://dx.doi.org/10.4314/mmj.v32i3.6 
Table 1: Baseline characteristics of patients presenting with signs and symptoms of an upper gastrointestinal bleed stratified by whether they received no endoscopy, diagnostic endoscopy, or endoscopy with variceal banding

\begin{tabular}{|c|c|c|c|c|}
\hline & $\begin{array}{l}\text { No Endoscopy } \\
(n=179)\end{array}$ & $\begin{array}{l}\text { Diagnostic Endoscopy } \\
\text { No Banding } \\
(n=65)\end{array}$ & $\begin{array}{l}\text { Endoscopy } \\
\text { With Banding } \\
(n=49)\end{array}$ & $p$ value \\
\hline \multicolumn{5}{|l|}{ Patient Age (years) } \\
\hline Mean (SD) & $41.6(16.1)$ & $43.7(17.0)$ & $40.2(12.6)$ & 0.08 \\
\hline \multicolumn{5}{|l|}{ Sex: N (\%) } \\
\hline Male & $113(63.1)$ & $42(64.6)$ & $25(51.0)$ & 0.51 \\
\hline \multicolumn{5}{|l|}{$\begin{array}{l}\text { Clinical History } \\
\text { Yes: } N(\%)\end{array}$} \\
\hline Had Previous Upper GI Bleed & $64(35.8)$ & $21(32.3)$ & $22(44.9)$ & 0.62 \\
\hline Ever Drank Alcohol Regularly & $72(40.2)$ & $26(40.0)$ & $13(26.5)$ & 0.41 \\
\hline Ever Smoked Tobacco Regularly & $50(27.9)$ & $17(26.2)$ & $7(14.3)$ & 0.26 \\
\hline Vomiting Blood & $173(96.7)$ & $63(96.9)$ & 47 (95.9) & 0.96 \\
\hline Black Stools & $116(64.8)$ & $45(69.2)$ & $35(71.4)$ & 0.77 \\
\hline \multicolumn{5}{|l|}{$\begin{array}{l}\text { Presenting Vital Signs: } \\
\text { Mean (SD) }\end{array}$} \\
\hline $\mathrm{HR}$ (bpm) & $100.0(19.6)$ & $95.4(16.8)$ & $97.7(25.0)$ & 0.029 \\
\hline $\mathrm{SBP}(\mathrm{mmHg})$ & $106.7(18.6)$ & $108.7(21.8)$ & $103.3(18.5)$ & 0.29 \\
\hline \multicolumn{5}{|l|}{ Presenting Labs: } \\
\hline $\begin{array}{l}\text { Hemoglobin Measured } \\
\text { Yes: } N(\%)\end{array}$ & $87(48.6)$ & $46(70.8)$ & $38(77.5)$ & $<0.001$ \\
\hline $\begin{array}{l}\text { Hemoglobin }(\mathrm{g} / \mathrm{dL}) \text { : } \\
\text { Mean (SD) }\end{array}$ & $7.2(3.3)$ & $8.1(4.1)$ & $6.2(2.2)$ & 0.001 \\
\hline $\begin{array}{l}\text { Platelet Level Measured } \\
\text { Yes: } \mathrm{N}(\%)\end{array}$ & $52(29.6)$ & $25(38.5)$ & $28(57.1)$ & 0.002 \\
\hline $\begin{array}{l}\text { Platelets }\left(\times 10^{9} / \mathrm{L}\right) \text { : } \\
\text { Mean (SD) }\end{array}$ & $133.7(123.0)$ & $253.9(214.6)$ & $91.5(111.3)$ & $<0.001$ \\
\hline \multicolumn{5}{|l|}{$\begin{array}{l}\text { Received Other Interventions } \\
\text { Yes: N (\%) }\end{array}$} \\
\hline $\begin{array}{l}\text { Packed Red Blood Cell Transfu- } \\
\text { sion }\end{array}$ & $120(67.0)$ & $47(72.3)$ & $42(85.7)$ & 0.12 \\
\hline Fresh Frozen Plasma Transfusion & $18(10.1)$ & $5(7.7)$ & $6(12.2)$ & 0.63 \\
\hline Platelet Transfusion & $3(1.7)$ & $2(3.1)$ & $0(0.0)$ & 0.26 \\
\hline Propranolol & $99(55.3)$ & $44(67.7)$ & $37(75.5)$ & 0.034 \\
\hline Proton Pump Inhibitor & $34(19.0)$ & $21(32.3)$ & $14(28.6)$ & 0.063 \\
\hline Spironolactone & $9(5.0)$ & $3(4.6)$ & $13(26.5)$ & $<0.001$ \\
\hline Diuretic & $30(16.8)$ & $20(30.8)$ & $12(24.5)$ & 0.050 \\
\hline \multicolumn{5}{|l|}{$\begin{array}{l}\text { Transfusion: Number of Units } \\
\text { Mean (SD) }\end{array}$} \\
\hline Packed Red Blood Cells & $2.1(1.4)$ & $2.2(1.4)$ & $2.8(1.4)$ & 0.91 \\
\hline \multicolumn{5}{|l|}{$\begin{array}{l}\text { Time to Transfusion Initiation } \\
\text { Yes: N }(\%)\end{array}$} \\
\hline Admission Day & $60(50.0)$ & $22(46.8)$ & $23(54.8)$ & 0.93 \\
\hline Day After Admission & $27(22.5)$ & $10(21.3)$ & $9(21.4)$ & \\
\hline Hospital Day 3 or Later & $33(27.5)$ & $15(31.9)$ & $10(23.8)$ & \\
\hline \multicolumn{5}{|l|}{ Time to Endoscopy (Days) } \\
\hline Mean (SD) & $\mathrm{n} / \mathrm{a}$ & $11.8(13.4)$ & $11.7(7.1)$ & 0.99 \\
\hline \multicolumn{5}{|l|}{ Outpatient Follow-up } \\
\hline \multicolumn{5}{|l|}{ Yes: N(\%) } \\
\hline Had Follow-up After Discharge & $56(31.3)$ & $22(33.9)$ & $11(22.5)$ & 0.39 \\
\hline
\end{tabular}




\section{table 1 cont....}

\author{
Had Repeat UGI Bleed \\ Death after Discharge \\ Crude Mortality
}

$$
\begin{aligned}
& \mathrm{N}(\%) \\
& \text { Died }
\end{aligned}
$$

\author{
$10(17.9)$ \\ $9(16.1)$
}

$3(13.6)$

$5(22.7)$

$4(36.4)$

$2(18.2)$

0.27

0.79

packed red blood cells (pRBC) or whole blood with only $29(9.9 \%)$ patients receiving a plasma transfusion and $5(1.7 \%)$ patients receiving a platelet transfusion. More patients in the banding group received a $\mathrm{pRBC}$ or whole blood transfusion compared to the other two groups $(67.0,72.3$, $85.7 \%, \mathrm{p}=0.12$ ) but each group had a similarly low proportion of patients that received either fresh frozen plasma or a platelet transfusion. Among those who received a $\mathrm{pRBC}$ or whole blood transfusion, the number of transfused units was similar (2.1 $\pm 1.4,2.2 \pm 1.4,2.8 \pm 1.4, \mathrm{p}=0.91$ ) despite significant differences in pre-transfusion hemoglobin levels. The timing of transfusion was also similar in each of the three groups with approximately half of patients in each group receiving their first transfusion on the day of admission (50.0\%, $46.8 \%, 54.8 \%, \mathrm{p}=0.93$ ). A quarter of patients received it on the day after admission $(22.5 \%, 21.3 \%, 21.4 \%)$ and the rest either on hospital day three or later $(27.5 \%, 31.9 \%, 23.8 \%)$. Propranolol was frequently used, especially in the endoscopy groups $(55.2 \%, 67.7 \%, 75.5 \%, \mathrm{p}=0.034)$ while omeprazole, spironolactone, and diuretics were used in less than $30 \%$ of patients.

We were able to follow-up post-discharge with relatively few patients in all three groups (Table 1; 31.3\%, 33.9\%, $22.5 \%, \mathrm{p}=0.39)$. Among those patients who had a follow-up, a higher proportion of patients in the banding group had suffered a repeat UGI bleed $(17.9 \%, 13.6 \%, 36.4 \%, \mathrm{p}=0.27)$. Follow-up mortality was comparable but high for all three intervention groups $(16.1 \%, 22.7 \%, 18.2 \%, \mathrm{p}=0.79)$.

Differences in mean time to endoscopy were not statistically significant in the two endoscopy groups, $11.8 \pm 13.4$ days vs. $11.7 \pm 7.1$ days, respectively (Table $1 ; \mathrm{p}=0.99$ ). Overall, only $11.1 \%$ of patients received endoscopy within three days of admission. Esophageal or gastric varices was the most common endoscopic diagnosis $(64.0 \%)$, with indeterminate $(14.9 \%)$ and gastric or duodenal ulcer $(7.9 \%)$ as the second and third most common diagnosis, respectively (Figure 1).

In-hospital crude mortality was significantly different between the three patient groups, $12.9 \%, 7.7 \%$, and $0.0 \%$, respectively $(\mathrm{p}=0.021$ ) (Table 1$)$. A first episode of bleeding and receiving at least one pRBC or whole blood transfusion were both significantly associated with increased

$5(7.7) \quad 0(0.0) \quad 0.021$

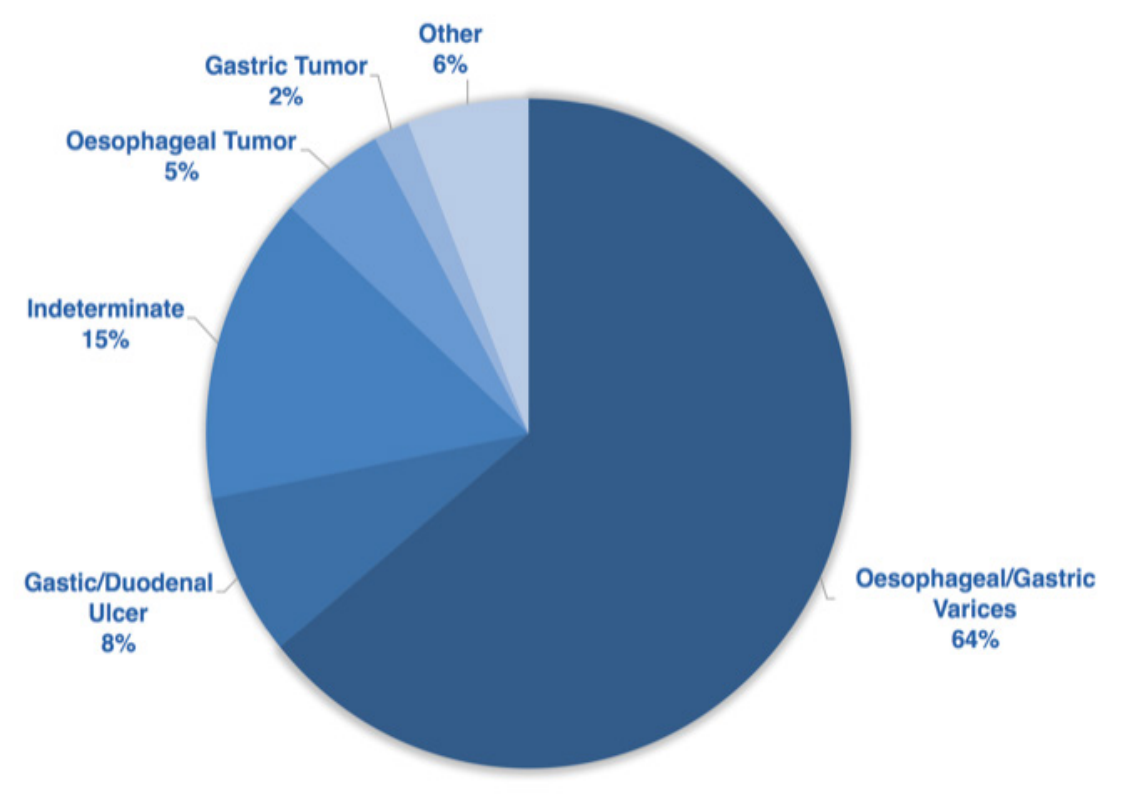

Figure 1: Endoscopic diagnosis of acute upper gastrointestinal bleeding for all patients who received endoscopy, with or without intervention

mortality. In addition, propranolol conferred a survival benefit with mortality rates of $6.7 \%$ and $14.2 \%$ in patients treated with and without propanol, respectively $(\mathrm{p}=0.034)$. Time to endoscopy, sex, alcohol use, presenting vital signs and laboratory studies, total units of pRBCs, fresh frozen plasma transfusion, spironolactone administration, and proton pump inhibitor administration were not significantly associated with an increase in mortality on bivariate analysis. Following imputation, an unadjusted Kaplan Meier survival analysis curve demonstrated a significant difference in the hazard of 30-day mortality among the three groups (Figure $2, \mathrm{p}=0.002)$. Cox hazard modeling showed a reduced hazard ratio of $0.12(95 \% \mathrm{CI} 0.02,0.88, \mathrm{p}=0.038)$ for the endoscopic banding group and a hazard ratio of 0.39 (95\% CI 0.13, 1.16 , $\mathrm{p}=0.090)$ for the diagnostic endoscopy group compared to the control group after adjusting for sex, propranolol administration, spironolactone administration, and whether the patient received a pRBC or whole blood transfusion.

We also examined relationships between patient characteristics, clinical history, and exam findings with an endoscopic diagnosis of variceal bleeding compared to those with a non-variceal endoscopic diagnosis. Mean age was significantly lower for those with a variceal diagnosis (39.6 \pm 13.1 years vs. $46.8 \pm 17.9$ years, $\mathrm{p}=0.017$ ) but sex was not 
different. There were more patients with a previous UGI bleed that had a variceal diagnosis $(76.4 \%$ vs. $57.8 \%, \mathrm{p}=0.039)$. Few patients had a platelet count among those who received endoscopy ( $\mathrm{n}=53$ ), but mean platelet count was significantly lower for those with a variceal diagnosis, $92.7 \pm 101.0 \times 10^{9} / \mathrm{L}$ vs. $359.1 \pm 213.8 \times 10^{9} / \mathrm{L}(\mathrm{p}<0.001)$. Physical exam findings consistent with cirrhosis or portal hypertension $(81.8 \%$ vs. $41.7 \%, \mathrm{p}<0.001)$ and ultrasound findings consistent with cirrhosis or portal hypertension ( $88.2 \%$ vs. $55.0 \%, \mathrm{p}=0.001)$ both had significant associations with a variceal diagnosis. Upon multivariate logistic regression modeling, positive physical exam findings consistent with cirrhosis and younger age were both independent predictors of an endoscopic diagnosis of variceal bleeding. Ultrasound findings were not included in the model as they were only available in a small subset of patients. Positive exam findings increased the odds of an endoscopic variceal diagnosis (OR 7.69, 95\% CI 3.10, $19.12, \mathrm{p}<0.001)$ while each ten-year increase in age decreased the odds of an endoscopic variceal diagnosis (OR 0.68, 95\% CI $0.51,0.91, \mathrm{p}=0.010)$. The predicted probability of varices varied widely depending on age and physical exam findings (Table 2).

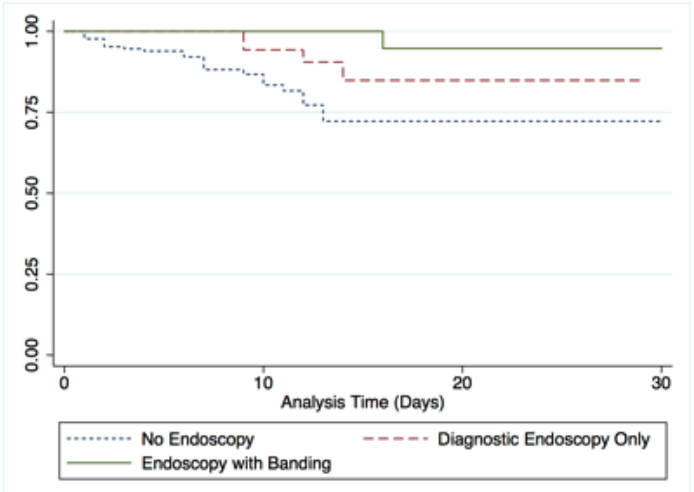

Figure 2: Kaplan-Meier Survival Estimates for 30Day In-Hospital Mortality stratified by whether they received no endoscopy, diagnostic endoscopy, or endoscopy with variceal banding, $\mathrm{p}=0.002$

For basic patient characteristics and clinical history, no variable was missing in more than $2 \%$ of patients. Vital signs were not documented in $8.9 \%$ of patients and physical exam findings were not documented for $22.9 \%$ of patients. We performed sensitivity analysis to assess any associations with missing vital sign or physical exam data by comparing patients with and without data.

There were no differences in patient characteristics, the intervention used, or mortality between patients with and without these data except that patients without documented clinical examination findings were less likely to receive endoscopy $(23.1 \%$ vs. $8.2 \%, p=0.034)$. The similarities between those with and without complete records suggest that these data were missing at random.

\section{Discussion}

The use of diagnostic or therapeutic endoscopy is critical to the management of patients with UGI bleeding regardless of setting. Limited endoscopy availability requires that providers in low-resource environments make informed patient triage decisions for intervention. At our center, like others throughout the region, it is not possible to offer every
Table 2: Adjusted predicted probability of varices based on patient age and physical exam findings. Probability reported with $95 \%$ confidence interval

\begin{tabular}{ll}
\hline Patients with & Patients without \\
Physical Exam & Physical Exam Findings \\
Findings & Consistent with Cirrhosis \\
Consistent with & or Portal Hypertension \\
Cirrhosis or & \\
Portal Hypertension & \\
\hline
\end{tabular}

Age (Years)

20

$0.92(0.81,0.97)$

$0.61(0.40,0.78)$

30

$0.89(0.78,0.95)$

$0.51(0.35,0.67)$

40

$0.84(0.73,0.92)$

$0.41(0.28,0.56)$

50

$0.79(0.66,0.88)$

$0.33(0.20,0.49)$

60

$0.72(0.54,0.84)$

$0.25(0.12,0.43)$

patient diagnostic or therapeutic endoscopy following UGI bleeding, despite the associated survival benefits.

In this study, we show that among patients presenting with acute UGI bleeding, endoscopy with variceal banding is lifesaving. In fact, over the two-year study period, no patient enrolled in the study died in the hospital after receiving banding. This was despite substantial delays in endoscopic intervention and limited blood transfusion services and pharmacotherapy. When adjusting for other commonly used therapies such as propranolol, the hazard ratio over thirty days was significantly lower for the endoscopy with banding group compared to no endoscopy. However, after adjustment, diagnostic endoscopy alone was not significantly associated with a decrease in the hazard of mortality compared to no endoscopy. While diagnosis is imperative for directing therapy, banding is the only endoscopic intervention available at our center. If patients did not receive banding, those in the diagnostic endoscopy group received the same pharmacotherapy as those who did not receive endoscopy. This similarity in medical therapy likely contributes to the similar hazard rates for the control group and the diagnostic endoscopy only group.

Consistent with data from the region, our study also highlights the high prevalence of variceal bleeding at our center with nearly two-thirds of patients in the endoscopy cohort having that diagnosis. ${ }^{4,5,7-11}$ We posit that because patients with bleeding varices have a survival benefit after banding, patients with likely varices based on clinical markers should be prioritized for endoscopy. Previous meta-analyses have shown a survival benefit along with decreased rates of re-bleeding and complications with endoscopy compared to medical therapy as well as the superiority of banding over sclerotherapy in most circumstances. ${ }^{14,15}$ Variceal bleeding is also less likely to stop spontaneously compared to nonvariceal bleeding. Less than $50 \%$ of variceal bleeding stops spontaneously compared to $80 \%$ of non-variceal bleeding. . $^{16,17}$ This study found that using age and physical exam findings together as predictors allows for simple triage of patients who are more likely to benefit from therapeutic endoscopy. Younger patients ( $<40$ years old) with physical exam findings consistent with cirrhosis had at least an $85 \%$ probability of varices in our model. While platelet count was not included in our model due to a small number of patients having laboratory studies available, a lower platelet count was 
significantly associated with varices and should likely be included in any triage algorithm if available. A previously published prediction model for bleeding in patients with esophageal varices included anatomical characteristics of varices as well as liver function tests rarely available for most patients here and not currently practical in our environment. ${ }^{18}$ As demonstrated in this study, post-procedure follow-up in this region is generally poor which limits data collection on the long-term efficacy of interventions. While there was no statistically significant mortality difference among the three groups, our data suggest there may be a higher proportion of patients with re-bleeding among those with variceal bleeding with $36 \%$ reporting a repeat bleeding episode after discharge. This is consistent with studies in South Africa that have shown that repeat bleeding is common among patients with liver cirrhosis after banding. ${ }^{19-22}$ Post-discharge mortality was also very high at almost $20 \%$ for all three groups and may be attributable to the underlying disease pathology such as liver cirrhosis. This highlights that variceal banding is ultimately a temporizing measure that attenuates in-hospital mortality and should be considered as a bridge to definitive therapy such as portosystemic shunting. Though transjugular intrahepatic portosystemic shunts (TIPS) are not available in our resource-poor setting, portacaval and splenorenal shunts can be performed.

In addition to appropriate triage for endoscopy, health care centers must work towards improving endoscopy capacity. Timely endoscopy was not available to all our patients who needed it due to staffing shortages of both physicians and nurses. Banding materials were also difficult to obtain. Also, patients did not receive appropriate blood transfusions and appropriate pharmacotherapies like somatostatin analogues and vasopressin which remain unavailable. Large scale endoscopy improvement projects and training programs have been successfully implemented in sub-Saharan Africa and warrant further consideration for centers without robust endoscopy programs. ${ }^{23-25}$

This study is limited by its observational methodology. Patients were not randomized to treatment thus exposing this study to possible selection bias. Overall, the three groups appear relatively similar and, with modeling, we attempted to control for potential confounders. However, in the cohort of patients that did not receive endoscopy, the diagnosis of esophagogastric varices is unknown. Therefore, the relative benefit of endoscopy in that cohort cannot be quantified. Additionally, we have limited laboratory data that could otherwise be valuable for risk assessment of patients. However, there are limited laboratory resources in our environment. Lastly, this study may be biased towards patients who survive a delay in treatment as many patients received endoscopic intervention several days after presentation.

\section{Conclusion}

Esophagogastric varices are a common cause of UGI bleeding in sub-Saharan Africa. In a resource-poor setting, its diagnosis can be predicted with age and physical exam findings. In addition, endoscopy with variceal banding has an in-hospital survival benefit for patients presenting with acute upper gastrointestinal bleeding even with relatively low utilization. Appropriately triaging patients with likely variceal bleeding and prioritizing the improvement of endoscopy capacity would likely have a significant impact on mortality.

\section{References}

1. Meara JG, Hagander L, Leather AJ. Surgery and global health: A Lancet Commission. Lancet. 2014;383(9911):12-13. doi:10.1016/ S0140-6736(13)62345-4.

2. Palmer KR. Non-variceal upper gastrointestinal haemorrhage: guidelines. Gut. 2002;51(suppl 4):iv1-6. doi:10.1136/gut.51.suppl_4. iv1.

3. Bendtsen F, Krag A, Møller S. Treatment of acute variceal bleeding. Dig Liver Dis. 2008;40(5):328-36. doi:10.1016/j.dld.2007.12.005.

4. Picrdo NG, Ajayi NA. Indications for an endoscopic findings in patients with symptoms of upper gastrointestinal disease in a Tertiary Hospital in South-Eastern Nigeria. Afr J Med Health Sci. 2015;14(2):96100. doi:10.4103/2384-5589.170169.

5. Sombié R, Tiendrébéogo A, Guingané A, Hagège H, Lesgourgues B, Lamy V, et al. Upper gastrointestinal bleeding: Epidemiological aspects and prognostic factors in Burkina Faso (Western Africa). J Afr Hepato Gastroenterol. 2015;9(4):154-159. doi:10.1007/s12157-015-0610-z.

6. Kayamba V, Sinkala E, Mwanamakondo S, Soko R, Kawimbe B, Amadi B, et al. Trends in upper gastrointestinal diagnosis over four decades in Lusaka, Zambia: a retrospective analysis of endoscopic findings. BMC Gastroenterol. 2015;15(1):127-135. doi:10.1186/ s12876-015-0353-8.

7. Mulima G, Qureshi JS, Shores C, Tamimi S, Klackenberg H, Andrén-Sandberg Å. Upper Gastrointestinal Bleeding at a Public Referal Hospital in Malawi. Surgical Science. 2014;5(11):501-507. doi:10.4236/ss.2014.511077.

8. Alema ON, Martin DO, Okello TR. Endoscopic findings in upper gastrointestinal bleeding patients at Lacor Hospital, northern Uganda. Afr Health Sci. 2013;12(4):518-521. doi:10.4314/ahs.v12i4.19

9. Suba M, Ayana SM, Mtabho CM, Kibiki GS. The aetiology, management and clinical outcome of upper gastrointestinal bleeding among patients admitted at the Kilimanjaro Christian Medical Centre in Moshi, Tanzania. Tanzan J Health Res. 2010;1(4):302-305. doi:10.4314/thrb.v12i4.51073

10. Mustapha SK, Ajayi N, Jibrin YB, Shehu A. Aetiology of Upper Gastrointestinal Bleeding in North-Eastern Nigeria: A Retrospective Review of Endoscopic Findings. Niger J Gastroenterol Hepato. 2009;1(2):75-78.

11. Kelly P, Katema M, Amadi B, Zimba L, Aparicio S, Mudenda V, et al. Gastrointestinal pathology in the University Teaching Hospital, Lusaka, Zambia: Review of endoscopic and pathology records. Trans R Soc Trop Med Hyg. 2008;102(2):194-199. doi:10.1016/j. trstmh.2007.10.006

12. Spearman CW, Sonderup MW. Health disparities in liver disease in sub-Saharan Africa. Liver Int. 2015;35(9):2063-2071. doi:10.1111/ liv. 12884

13. Makaula P, Sadalaki JR, Muula AS, Kayuni S, Jemu S, Bloch P. Schistosomiasis in Malawi: A systematic review. Parasit Vectors. 2014;7(1):570-590. doi:10.1186/s13071-014-0570-y

14. De Franchis R, Primignani M. Endoscopic treatments for portal hypertension. Semin Liver Dis. 1999;19(4):439-455. doi:10.1055/s-2007-1007131

15. Laine L, Cook D. Endoscopic ligation compared with sclerotherapy for treatment of esophageal variceal bleeding: a meta-analysis. Ann Intern Med. 1995;123(4):280-287.

16. Augustin S, González A, Genescà J. Acute esophageal variceal bleeding: Current strategies and new perspectives. World J Hepatol. 2010;2(7):261-274. doi:10.4254/wjh.v2.i7.261

17. Palmer K. Acute upper gastrointestinal haemorrhage. Br Med Bull. 2007;83(1):307-324. doi:10.1093/bmb/ldm023

18. North Italian Endoscopic Club for the Study and Treatment of 
Esophageal Varices. Prediction of the First Variceal Hemorrhage in Patients with Cirrhosis of the Liver and Esophageal Varices. N Engl J Med. 1988;319(15):983-989. doi:10.1056/NEJM198810133191505

19. Krige JE, Kotze UK, Bornman PC, Shaw JM, Klipin M. Variceal recurrence, rebleeding, and survival after endoscopic injection sclerotherapy in 287 alcoholic cirrhotic patients with bleeding esophageal varices. Ann Surg. 2006;244(5):764-770. doi:10.1097/01. sla.0000231704.45005.4e

20. Krige JE, Bornman PC, Goldberg PA, Terblanche J. Variceal rebleeding and recurrence after endoscopic injection sclerotherapy: A prospective evaluation in 204 patients. Arch Surg. 2000;135(11):13151322. doi:10.1097/01.sla.0000231704.45005.4e

21. Krige JEJ, Shaw JM, Bornman PC, Kotze UK. Early rebleeding and death at 6 weeks in alcoholic cirrhotic patients with acute variceal bleeding treated with emergency endoscopic injection sclerotherapy. S Afr J Surg. 2009;47(3):72-79.

22. Varghese J, Cherian JV, Solomon R, Jayanthi V. Predictors of variceal bleed among patients with liver cirrhosis in the era of sclerotherapy. Singapore Med J. 2008;49(3):239-242.
23. Asombang AW, Turner-Moss E, Seetharam A, Kelly P. Gastroenterology training in a resource-limited setting: Zambia, Southern Africa. World J Gastroenterol. 2013;19(25):3996-4000. doi:10.3748/wjg.v19.i25.3996

24. Le Moine O, Diouf M, Mbengue M, Mbaye P, Diop P, Balme F, et al. Creation of a therapeutic digestive endoscopy suite in Senegal: renovation, training and university certification. Results of a BelgianSenegalese inter-university project. Endoscopy. 2012;44(02):177-185. doi:10.1055/s-0031-1291584

25. Ladep NG, Sule J, Umar SM, Obienu O, Anyanechi C, Okeke EN. Oesophageal variceal band ligation using a saeed six-shooter multiband ligator; experience at Jos University Teaching Hospital, Nigeria: case report. Niger J Med. 2008;17(1):110-111. doi:10.4314/ njm.v17i1.37368 